\title{
Fast-electron-relaxation measurement for laser-solid interaction at relativistic laser intensities
}

\author{
H. Chen, ${ }^{1}$ R. Shepherd, ${ }^{1}$ H. K. Chung, ${ }^{1}$ A. Kemp, ${ }^{1}$ S. B. Hansen, ${ }^{1}$ S. C. Wilks, ${ }^{1}$ Y. Ping, ${ }^{1}$ K. Widmann, ${ }^{1}$ K. B. Fournier, ${ }^{1}$ \\ G. Dyer, ${ }^{2}$ A. Faenov, ${ }^{3}$ T. Pikuz, ${ }^{3}$ and P. Beiersdorfer ${ }^{1}$ \\ ${ }^{1}$ Physics and Advanced Technologies, Lawrence Livermore National Laboratory, \\ University of California, Livermore, California 94550, USA \\ ${ }^{2}$ Department of Physics, University of Texas, Austin, Texas 78712, USA \\ ${ }^{3}$ Multicharged Ion Spectra Data Center, VNIIFTRI, Mendeleevo, Moscow oblast, 141570 Russia \\ (Received 14 May 2007; revised manuscript received 25 September 2007; published 14 November 2007)
}

\begin{abstract}
We present measurements of the fast-electron-relaxation time in short-pulse $(0.5 \mathrm{ps})$ laser-solid interactions for laser intensities of $10^{17}, 10^{18}$, and $10^{19} \mathrm{~W} / \mathrm{cm}^{2}$, using a picosecond time-resolved $\mathrm{x}$-ray spectrometer and a time-integrated electron spectrometer. We find that the laser coupling to hot electrons increases as the laser intensity becomes relativistic, and that the thermalization of fast electrons occurs over time scales on the order of $10 \mathrm{ps}$ at all laser intensities. The experimental data are analyzed using a combination of models that include $K \alpha$ generation, collisional coupling, and plasma expansion.
\end{abstract}

DOI: 10.1103/PhysRevE.76.056402

PACS number(s): 52.50.Jm, 52.38.Ph, 32.30.Rj

\section{INTRODUCTION}

It is well established that high-intensity lasers incident on solid targets can generate relativistic electrons $[1,2]$ through interactions with the laser electric field or the associated ponderomotive force. These hot electrons can effectively heat the solid target beyond the region of direct laser interaction. This is an important aspect of the fast ignition concept, part of a major effort to generate controlled thermonuclear fusion [3]. How hot electrons couple to the bulk solid target is an important open question, since many applications rely on assumed high coupling and rapid thermalization of hot electrons to background thermal electrons. In fast ignition, for example, the thermalization time must be shorter than the time for the compressed core to hydrodynamically disassemble, which is currently thought to be less than about 20 ps [3].

$K \alpha$ inner-shell radiation, generated when hot electrons excite or ionize the core electrons of atoms in the solid, provides a diagnostic for the presence of hot electrons. In fact, measurements of absolute $K \alpha$ radiation intensity are commonly used to estimate the number of hot electrons generated during the complex laser-target interaction [4-8]. Although the time history of $K \alpha$ emission has been previously measured for laser intensities lower than $10^{17} \mathrm{~W} / \mathrm{cm}^{2}$ [9-12], $K \alpha$ measurements at relativistic laser intensities have up to now all been time integrated and have thus given no insight into the time scale of the hot-electron coupling to bulk thermal electrons. The direct, time-resolved measurement of the $K \alpha$ signal presented here gives a direct determination of the coupling time, significantly increasing our understanding of the physical process of hot-electron relaxation.

\section{DESCRIPTION OF EXPERIMENT AND RESULTS}

The experiment was performed at the Compact Multipulse Terawatt (COMET) laser facility [13] at Lawrence Livermore National Laboratory. COMET is a hybrid chirped pulse amplification (CPA) system with a Ti:sapphire oscillator and regenerative amplifier with a four-stage Nd:phos- phate glass amplifier. The laser wavelength is $1054 \mathrm{~nm}$. For the data presented in this paper, the laser pulse was $p$ polarized with a length of $500 \mathrm{fs}$ at full width at half maximum (FWHM). The laser energy (6-7 J) was focused with an f/3.6 parabola onto a solid target at an incident angle of $45^{\circ}$. The best focus was typically $8-10 \mu \mathrm{m}$ FWHM, resulting in a laser intensity of $10^{19} \mathrm{~W} / \mathrm{cm}^{2}$ at the best focus. By systematically increasing the spot size on the target, we were able to decrease the laser intensity down to $10^{17} \mathrm{~W} / \mathrm{cm}^{2}$ while keeping the laser energy constant.

Prepulse is a major concern in short-pulse, laser-solid experiments. The temporal intensity contrast of the pulses is characterized by three components: (1) a 12 ns prepulse produced by leakage in the regenerative amplifier, (2) amplified spontaneous emission (ASE), and (3) a pedestal produced by the third- and fourth-order aberrations in the stretcher. Often, the most significant prepulse comes from the leakage pulse from the regenerative amplifier. Using two Pockels cells and polarizers, a contrast of $I_{\text {leakage }} / I_{\text {main }} \approx 10^{-9}$ was obtained. No measurement was made of the contribution of ASE or thirdand fourth-order aberrations. However, an analysis of the spectrum from the stretcher-compressor system suggests the contribution of the third- and fourth-order aberrations to nonGaussian components produce a contrast of $I_{\text {aberr }} / I_{\text {main }}$ $\approx 10^{-5}$ at about 2 ps before the peak of the pulse [14]. To confirm that the ASE level produced no preformed plasma, solid targets were illuminated without seeding the amplifiers with the oscillator pulse. No damage was observed, suggesting minimal effect from ASE. As a monitor of the preformed plasma, a visible spectrometer was set up to look at the specular reflected laser light. Because Raman scattering and two-plasmon decay are a strong function of density scale length [15], the spectrally resolved, specular light provided a shot-to-shot monitor of large fluctuations in the prepulse. We observed mostly constant harmonic spectra throughout the experiments, indicating stable preplasma conditions during the laser-target interactions.

The laser targets consisted of $12.5 \mu \mathrm{m}$ of Ti overcoated with $1000 \AA$ of Al. The Al layer prevented direct illumination of the Ti foil, thus eliminating any direct laser heating. The $\mathrm{x}$-ray emission from the target was collected using a 


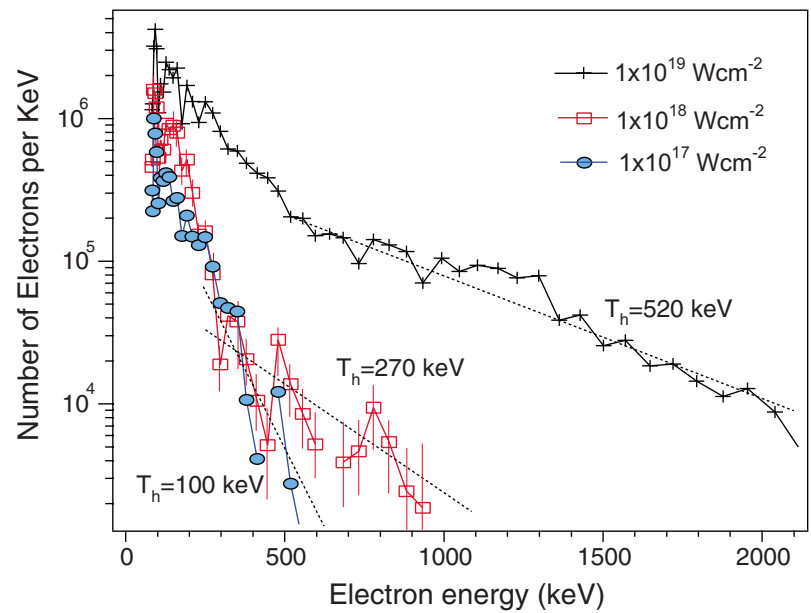

FIG. 1. (Color online) Electron spectroscopy data from FACES for the three laser intensities. Error bars of the measurement are marked for the medium intensity. The dotted lines are the exponential fit to the high-energy section of the spectra.

time-resolved x-ray streak camera [16] interfaced to two crystals arranged in the von Hàmos geometry: a graphite crystal with an $11 \mathrm{~cm}$ radius of curvature $\left(R_{c}\right)$ to collect the Ti $K \alpha$ emission and a second crystal with $R_{c}=3 \mathrm{~cm}$ to collect the Al $K$-shell $1 s 2 p\left({ }^{1} P\right)-1 s^{2}\left({ }^{1} S\right)(\mathrm{He}-\alpha)$ emission. The streak camera was coupled to an image intensifier, a 2:1 fiber optic reducer, and a fiber optic face plate mounted on a 16bit, water-cooled, $1024 \times 1024$ pixel charge-coupled device. The time resolution of the streak camera is about $1 \mathrm{ps}$, and the total coverage is about 40 ps. A time-integrated measurement of the electrons escaping the target was made using an absolutely calibrated fiber-optic array compact electron spectrometer (FACES) [17], which was set up to measure electrons from $80 \mathrm{keV}$ to $6 \mathrm{MeV}$. It was positioned $37 \mathrm{~cm}$ behind the target, $15^{\circ}$ off the laser propagation direction with a solid angle of $1 \times 10^{-4}$ steradians. Finally, a time-integrated, spatially resolved $\mathrm{x}$-ray spectrometer with a spherically bent quartz crystal was fielded to view the back surface of the target, providing high-resolution spectra from which the heating of the Ti target was determined [18]. This diagnostic also measured the transversal size of the emission region on the back side of Ti targets, which showed that the emission region actually increases with decreasing focal spot size (increasing laser intensities).

Data were collected at laser focal intensities of $10^{17}, 10^{18}$, and $10^{19} \mathrm{~W} / \mathrm{cm}^{2}$, spanning the transition from nonrelativistic $\left[P_{\mathrm{os}} /\left(m_{e} c\right)=0.3\right]$ to relativistic $\left[P_{\mathrm{os}} /\left(m_{e} c\right)=3\right]$ laser intensities, where $P_{\mathrm{os}}$ is the electron momentum and $m_{e}$ the electron mass. Figure 1 shows that significantly more hot electrons were observed by the electron spectrometer as the laser intensity became relativistic: the number of $\approx 100 \mathrm{keV}$ electrons per keV increases from $4 \times 10^{5}$ for $I=10^{17}$, to $9 \times 10^{5}$ for $I=10^{18}$, and to $2 \times 10^{6}$ for $I=10^{19} \mathrm{~W} / \mathrm{cm}^{2}$. This increase is consistent with the measured enhanced laser absorption at higher laser intensities described in Ref. [19]. A quantitative description of the hot-electron energy distribution is the socalled hot-electron temperature $T_{h}$, obtained by fitting the spectrum with one or more quasi-Maxellian distribution functions. To estimate $T_{h}$ right after the laser pulse from the time-integrated spectra, we fit a Maxwellian to the high end of the energy distribution, reasoning that the hot-electron energy can only decrease after the laser pulse is turned off. Fits to the high end of the electron distribution give $T_{h}=100,270$, and $520 \mathrm{keV}$ for laser intensities of $10^{17}, 10^{18}$, and $10^{19} \mathrm{~W} / \mathrm{cm}^{2}$, respectively. These temperatures agree approximately with the ponderomotive scaling of hot electrons [20] except for the highest intensity, where the measured $T_{h}$ is about half of the predicted value.

The angular dependence of the hot electron distributions from short-pulse solid interactions has been determined experimentally and explained theoretically [19,21-27]. For our case (a high-contrast $p$-polarized laser incident at $45^{\circ}$ to a foil target), the most dramatic angular variations would be due to collimated hot-electron jets. At intensities of $10^{17}$ and $10^{18} \mathrm{~W} / \mathrm{cm}^{2}$, these jets form mostly in the specular and target normal directions at the front of the target $[22,27,28]$ and are driven by resonant absorption. At $10^{19} \mathrm{~W} / \mathrm{cm}^{2}$, another jet forms at the back of the target due to poderomotive heating. The angle of this jet is predicted to be about $20^{\circ}$ off the laser direction [26,27]. Since the FACES measurement looks at the back of the target $15^{\circ}$ off the laser direction, it is not in the path of the possible jets. Based on the studies of Refs. [22,26-28], we estimate the FACES measurements to be within $10-35 \%$ of the $2 \pi$ average of any plausible anisotropic distribution.

Although the large majority of hot electrons at the measured $T_{h}$ pass unperturbed through the thin Al layer on the front of the target, the $1000 \AA \mathrm{Al}$ is heated by both direct interactions with the incident laser and the laser-generated hot electrons. Measurements of $\mathrm{Al} K$-shell emission from the TREX spectrometer at wavelengths between 7.6 and $7.9 \AA$ indicate significant heating but little expansion of the $\mathrm{Al}$ layer: We observed a prominent $\mathrm{Al} \mathrm{He}-\alpha$ line at $7.765 \AA$, but detected neither a strong intercombination line $\left[1 s^{2}\left({ }^{1} S\right)-1 s 2 p\left({ }^{3} P\right)\right]$ at $7.806 \AA$ nor significant satellite emission from Li-like or lower-charged $\mathrm{Al}$ ions. The absence of the density-sensitive intercombination line (whose upper level is a metastable state that is collisionally depopulated at densities higher than $10^{21} \mathrm{~cm}^{-3}$ [29]) indicates that no significant preplasma is formed before the arrival of the main pulse, confirming the prepulse and Raman measurements discussed above.

Figure 2 shows the time history of the measured Ti $K \alpha$ intensities for the three laser intensities. We measure the hotelectron relaxation time (defined as the FWHM of $I_{K \alpha}$ ) to be $15.9,13.2$, and $12.3 \mathrm{ps}$, for laser intensities of $10^{19}, 10^{18}$, and $10^{17} \mathrm{~W} / \mathrm{cm}^{2}$, respectively. These $K \alpha$ emission durations are significantly larger than those measured previously [9-12]. This is due to the fact that our hot-electron temperatures are at least one order of magnitude larger than in previous experiments; as a consequence, the hot-electron range is many times the target thickness so that multiple interaction and $K \alpha$ generation can occur with parts of the target that are transparent for $K \alpha$ radiation.

In addition to generating $K \alpha_{1}$ emission through collisions with $\mathrm{Ti}$ ions, the hot electrons can increase the temperature of the bulk target through collisions with bulk (thermal) electrons. An analysis of spherical crystal spectrometer measurements indicated a final bulk temperature $T_{c} \approx 50 \mathrm{eV}$ and an 
average $\mathrm{Ti}$ ion charge of $4-5$ [18]. The Al layer is also heated partially by the hot electrons and remains hot enough to emit significant $\mathrm{Al} \mathrm{He}-\alpha$ radiation long after the Ti $K \alpha$ emission has decayed. The $\mathrm{Al} \mathrm{He}-\alpha$ emission persists over at least $40 \mathrm{ps}$ (the time coverage of the streak camera).

\section{ANALYSIS AND MODELING}

Shielded by the Al layer from direct laser heating, the Ti layer mostly interacts with hot electrons that excite and ionize $K$-shell electrons and produce Ti $K \alpha$ emission. Because the decay of the upper $(n=2)$ level is extremely rapid, occurring in about 5 fs for Ti $K \alpha_{1}$ [30], the Ti $K \alpha$ intensity from each ion is limited by the rate of electron-impact ionization from the $1 s$ shell: $n_{e}\left\langle F(E) v_{e} \sigma(E)\right\rangle s^{-1}$, with $F(E)$ a normalized hot-electron distribution function, $v_{e}$ the impact electron velocity, and $\sigma(E)$ the cross section of electron impact ionization. The $K \alpha$ radiation emitted from a volume element $d V$ of ions with density $n_{i o n}$ is thus

$$
I_{K \alpha}^{(1)}=\int_{V, E} n_{\mathrm{ion}} n_{e} F(E) v_{e}(E) \sigma(E) d V d E \quad \text { (photons/s). }
$$

The total $K \alpha$ intensity is typically obtained by integrating $n_{\text {ion }}$ over $d V$ and summing $I_{K \alpha}^{(1)}$ over volume elements with various electron distributions. However, since the integration over $F(E)$ is limited to those electrons with energies greater than $\Delta E \approx 5 \mathrm{keV}$, the number of hot electrons rather than the number of emitting ions is the limiting factor on the $K \alpha$ intensity and we may integrate over $n_{e}$ rather than $n_{\text {ion. }}$. (For example, the total $K \alpha$ intensity from a $10-\mu$ m-diameter region with a hot-electron fraction $f=10^{-1}$ would be the same as that from a $100 \mu \mathrm{m}$ region with $f=10^{-3}$.) Holding $n_{\text {ion }}$ constant at each moment and integrating the hot-electron density over all space, we thus obtain

$$
I_{K \alpha}(t)=N_{h}(t) n_{\text {ion }}(t) \int_{E>\Delta E} F(E, t) \sigma(E) v_{e}(E) d E \quad \text { (photons/s). }
$$

This intensity is dependent on the number of hot electrons $N_{h}$, the ion density, and (weakly) $F(E ; E>\Delta E \approx 5 \mathrm{keV})$. It is important that $I_{K \alpha}(t)$ is independent of the emission volume, so we need not know the time evolution of the spatial distribution of hot electrons.

To model the time-dependent $K \alpha$ emission and interpret the measured hot-electron relaxation times, we have developed an expanding multicomponent plasma model. In this model, hot electrons initially injected into the target at a temperature $T_{h 0}$ can lose energy in two ways: (i) through direct collisions with bulk electrons that transfer energy from the hot electrons to cold electrons and ions and (ii) through adiabatic cooling due to plasma expansion as determined by the ion sound speed [31]. Since the hot electrons move much faster than the ions $\left(v_{i}<0.1 c\right.$ for protons typically, while $\left.v_{e} \approx c\right), T_{h}$ evolves adiabatically according to $p V^{\gamma}=$ const. The volume $V=L^{d}$ is defined by the ion-front position and the geometry of the expansion, which can be planar $(d=1)$, as assumed here, or spherical $(d=3)$, which might apply to

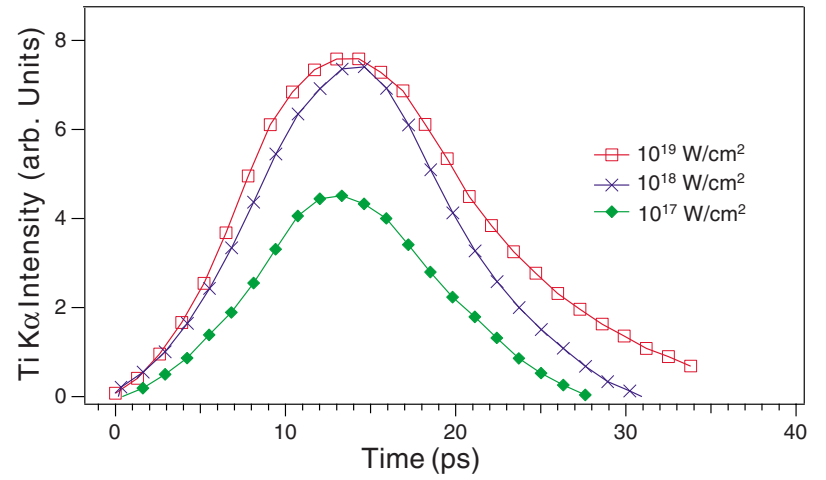

FIG. 2. (Color online) Ti $K \alpha$ line intensity as a function of time at the three laser intensities. The full width at half maximum is 15.9, 13.2 , and $12.3 \mathrm{ps}$ measured at laser intensities of $1 \times 10^{19}, 1$ $\times 10^{18}$, and $1 \times 10^{17} \mathrm{~W} / \mathrm{cm}^{2}$, respectively.

thin targets with tightly focused laser foci. The combination of expansion and collisions gives

$$
\begin{gathered}
\frac{d T_{h}}{d t}=\left(\frac{L(t)}{L_{0}}\right)^{-d} \times \nu_{\epsilon}^{h c}\left(T_{c}-T_{h}\right)+d(1-\gamma) \\
\times T_{h 0}\left(\frac{L(t)}{L_{0}}\right)^{-d \gamma}\left(\frac{L(t)}{L_{0}}\right)^{d-1} \frac{d L(t)}{d t}, \\
\frac{d T_{c}}{d t}=\left(\frac{L(t)}{L_{0}}\right)^{-d} \times \nu_{\epsilon}^{c h}\left(T_{h}-T_{c}\right), \\
\frac{d L(t)}{d t}=2 c_{s}(t)\left[2 \ln \left(\Omega_{i} t+1\right)+\ln 2-1\right],
\end{gathered}
$$

where $\nu_{\epsilon}^{h c}$ is the energy transfer rate between hot and cold electrons given by

$$
\nu_{\epsilon}^{h c}=\frac{8 \sqrt{2 \pi} e^{4}}{3 \sqrt{m_{e}}} \frac{n_{c} \ln \Lambda}{\left(k_{B} T_{c}+k_{B} T_{h}\right)^{3 / 2}} .
$$

Here the ion sound velocity $c_{s}(t)=\sqrt{T_{h}(t) / M_{i}}$ and $\Omega_{i}$ $=\sqrt{4 \pi n_{i} Z^{2} e^{2} / M_{i}}$ is the plasma frequency for ions with density $n_{i}$, mass $M_{i}$, and charge $Z[32]$. The factor $\left[L(t) / L_{0}\right]^{-d}$ enters Eqs. (3) and (4) to account for the fraction of the time that the hot electrons spend inside the target. We use an adiabatic coefficient of $\gamma=5 / 3$ and a Coulomb logarithm of $\ln \Lambda=5$. Electron-ion collisions are omitted because they contribute only a fraction $Z^{2}\left(m_{e} / m_{i}\right) \ll 1$ to collisional relaxation.

The predictions of this simple relaxation model under conditions that are roughly comparable to the experiment for a laser intensity of $10^{19} \mathrm{~W} / \mathrm{cm}^{2}$ are shown in the inset in Fig. 3. With $T_{h}=500 \mathrm{keV}$, our model predicts an initial drop in $T_{h}$ over the first $\approx 5$ ps due to expansion and equilibration after $\approx 20$ ps due to collisions. The final bulk temperature of a few tens of $\mathrm{eV}$ is in good agreement with the bulk temperature determined by spectroscopic measurements [18]. The role of expansion is most important for large $T_{h}$ values: neglecting expansion in our example case with $T_{h}=500 \mathrm{keV}$ extends the equilibration time to $30 \mathrm{ps}$, but has no noticeable effect for $T_{h} \approx 100 \mathrm{keV}$. 


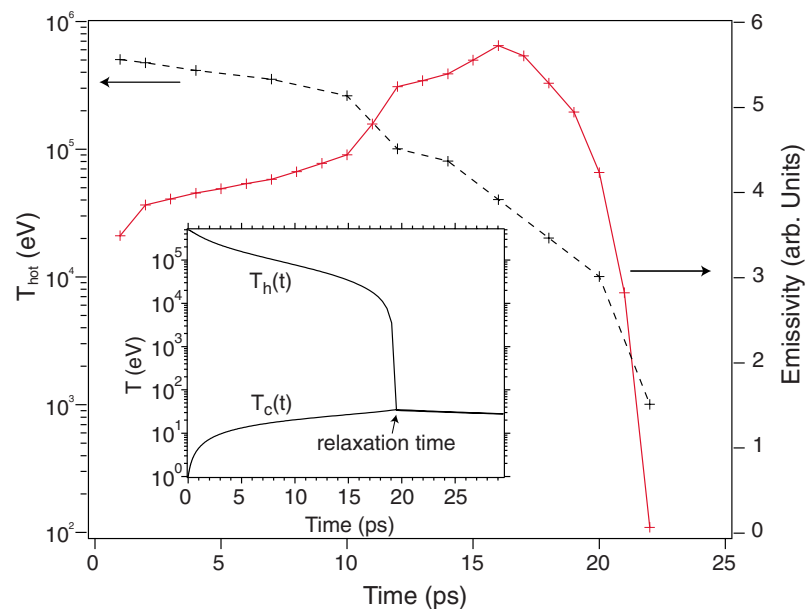

FIG. 3. (Color online) Calculated total Ti $K \alpha$ emissivity (solid line) as a function of time. The hot-electron temperature used (dashed line) was derived from the experiment. The inset shows the evolution of hot- and cold-electron temperatures as functions of time for a solid density $\mathrm{Ti}^{4+}$ plasma with $n_{\text {hot }}=10^{20} \mathrm{~cm}^{-3}$.

We have used the measured hot electron temperature and the relaxation profile from the above model with the collisional-radiative code FLYCHK [33] to derive the Ti $K \alpha$ profile under the assumption that the $K \alpha$ signal is due solely to laser-generated hot electrons. FLYCHK determines timedependent screened hydrogenic level populations by solving a coupled set of rate equations which include collisional excitation, deexcitation, ionization, and recombination as well as radiative emission, recombination, electron capture, and autoionization processes. These level populations are used with Eqs. (1) and (2) to determine the $K \alpha$ signal as a function of time. As shown in Fig. 3 for the case of $10^{19} \mathrm{~W} / \mathrm{cm}^{2}$, the time duration $(10-20 \mathrm{ps})$ of the modeled $K \alpha$ is close to that measured by the experiment (15.9 ps). For the two lower laser intensities, the agreement between modeled $K \alpha$ duration $(2-10 \mathrm{ps})$ and the measurements $(12-13 \mathrm{ps})$ is less satisfactory. Moreover, even when the model matches the decay of the $K \alpha$ intensities, it does not reproduce the measured rise. Unlike earlier studies at lower laser intensities $\left(10^{14}-10^{17} \mathrm{~W} / \mathrm{cm}^{2}\right)$ [10-12] where the measured $K \alpha$ burst time was close to the laser duration, the main pulse (500 fs) and $K \alpha$ signal (10-15 ps) cannot be coincident in our experiment.

Assuming that the main laser pulse occurs before the maximum $K \alpha$ intensity and that the hot electrons spread throughout the target thickness rather than remaining in a thin surface layer, there are two possibilities which can account for the few-picosecond measured rise in $K \alpha$. The first is that the early $K \alpha$ signal is due to hot electrons generated well before the peak of the main laser pulse. In this scenario, the $K \alpha$ emission rises along with a prepulse laser intensity, reaching its maximum near the time of the peak intensity of the main pulse. While a non-Gaussian component due to imperfections in the recompression could in principal contribute to the population of $K \alpha$-generating electrons before the peak of the main pulse, with our laser contrast we do not expect the prepulse to produce measurable $K \alpha$.

The second, more plausible, scenario is that the main pulse occurs well before the $K \alpha$ intensity peak, creating an initial population of hot electrons that both directly generates $K \alpha$ photons via inner-shell processes and creates secondary " $K \alpha$ electrons" (i.e., electrons with sufficient energy to produce further inner-shell ionization) via ionization of valence ( $M$-shell) electrons. To quantify this scenario, we have computed the energy distribution of secondary electrons created in an $M$-shell ionization event. We find that the fraction of secondary electrons with $E>5 \mathrm{keV}$ is more than $30 \%$ for impact electron energies of $100 \mathrm{keV}$. This fraction increases with the impact electron energy. By comparing the rates of $M$-shell and $K$-shell ionization processes for the hot electrons, we find that for each electron with energy $100-300 \mathrm{keV}, M$-shell ionization is $400-500$ times more likely than $K$-shell ionization. Thus, for each collision of a hot electron with an $M$-shell ion, direct production of $K \alpha$ emission has a probability of less than $1 \%$ while production of a secondary electron which can itself produce $K \alpha$ emission is around $30 \%$. For an initial hot-electron fraction of $10^{-3}$ (an estimate obtained by assuming that $50 \%$ of the laser energy goes into hot electrons in a $12.5 \mu \mathrm{m}$ by $200 \mu$ m-diameter disk in the solid Ti target), the $M$-shell ionization rate is $\approx 10^{12} \mathrm{~s}^{-1}$. The resulting time scale of secondary electron generation is a few picoseconds, which corresponds rather well to the observed $K \alpha$ risetime. The number of secondary electrons is limited by energy conservation to be $10-20$ for each initial $\approx 100 \mathrm{keV}$ electron produced by the main laser pulse. In this scenario, the observed risetime of $K \alpha$ (and indeed the majority of the $K \alpha$ production) is due to an effective increase in the value of $N_{h}$ given in Eq. (2) not through direct excitation by either the main laser pulse or a prepulse, but through valence-shell ionization processes occurring on the same time scale as the temperature thermalization.

\section{SUMMARY}

We have measured the relaxation time of hot electrons produced in short-pulse laser-solid interactions at relativistic laser intensities, advancing our understanding of fast electron transport and coupling for fast ignition and other applications. We find increased laser coupling to hot electrons as the laser intensity becomes relativistic and a much longer (on the order of $10 \mathrm{ps}$ ) duration of $K \alpha$ emission than the temporal duration of the main laser pulse. The time scale observed $(12-16 \mathrm{ps})$ is still short enough to allow laser-driven hot electrons to be useful for fast-ignitor energy deposition. We have proposed a simple model based on collisional coupling and plasma expansion. We also discussed the possible effect on the time history of $K \alpha$ from secondary $K \alpha$ electron generation from the interaction between the primary hot electrons and the target.

\section{ACKNOWLEDGMENTS}

This work was performed under the auspices of the U.S. Department of Energy by University of California Lawrence Livermore National Laboratory under Contract No. W-7405Eng-48. 
[1] M. H. Key et al., Phys. Plasmas 5, 1966 (1998).

[2] S. P. Hatchett et al., Phys. Plasmas 7, 2076 (2000).

[3] M. Tabak et al., Phys. Plasmas 1, 1626 (1994).

[4] K. B. Wharton, S. P. Hatchett, S. C. Wilks, M. H. Keys, J. D. Moody, V. Yanovsky, A. A. Offenberger, B. A. Hammel, M. D. Perry, and C. Joshi, Phys. Rev. Lett. 81, 822 (1998).

[5] R. A. Snavely et al., Phys. Rev. Lett. 85, 2945 (2000).

[6] F. Pisani et al., Phys. Rev. E 62, R5927 (2000).

[7] C. Reich, I. Uschmann, F. Ewald, S. Düsterer, A. Lübcke, H. Schwoerer, R. Sauerbrey, E. Förster, and P. Gibbon, Phys. Rev. E 68, 056408 (2003).

[8] D. Riley, J. J. Angulo-Gareta, F. Y. Khattak, M. J. Lamb, P. S. Forster, E. J. Divall, C. J. Hooker, A. J. Langley, R. J. Clarke, and D. Neely, Phys. Rev. E 71, 016406 (2005).

[9] U. Andiel, K. Eidmann, K. Witte, I. Uschmann, and E. Förster, Appl. Phys. Lett. 80, 198 (2002).

[10] Ch. Reich, P. Gibbon, I. Uschmann, and E. Förster, Phys. Rev. Lett. 84, 4846 (2000).

[11] E. Pisani, U. Andiel, K. Eidmann, K. Witte, I. Uschmann, A. Morak, E. Förster, and R. Sauerbrey, Appl. Phys. Lett. 84, 2772 (2004).

[12] T. Feurer, A. Morak, I. Uschmann, C. Ziener, H. Schwoerer, C. Reich, P. Gibbon, E. Förster, R. Sauerbrey, K. Ortner, and C. R. Becker, Phys. Rev. E 65, 016412 (2001).

[13] J. Dunn et al., Opt. Lett. 24, 101 (1999).

[14] J. Dunn et al., in Laser Interaction and Related Plasma Phenomena, edited by S. Nakai and G. H. Miley, AIP Conf. Proc. No. 369 (AIP, Woodbury, NY, 1996), pp. 652-659.

[15] C. S. Liu and M. N. Rosenbluth, Phys. Fluids 19, 967 (1976); L. Veisz, W. Theobald, T. Feurer, H. Schwoerer, I. Uschmann, O. Renner, and R. Sauerbrey, Phys. Plasmas 11, 3311 (2004).
[16] R. Shepherd et al., Rev. Sci. Instrum. 75, 3765 (2004).

[17] H. Chen et al., Rev. Sci. Instrum. 74, 1551 (2003).

[18] S. B. Hansen, A. Y. Faenov, T. A. Pikuz, K. B. Fournier, R. Shepherd, H. Chen, K. Widmann, S. C. Wilks, Y. Ping, H. K. Chung, A. Niles, J. R. Hunter, G. Dyer, T. Ditmire, Phys. Rev. E 72, 036408 (2005).

[19] Y. Ping et al., Univerisity of California, Lawrence Livermore National Laboratory Report No. UCRL-JRNL-219398, 2006 (unpublished).

[20] S. C. Wilks, W. L. Kruer, M. Tabak, and A. B. Langdon, Phys. Rev. Lett. 69, 1383 (1992).

[21] G. Malka and J. L. Miquel, Phys. Rev. Lett. 77, 75 (1996).

[22] D. F. Cai et al., Phys. Plasmas 10, 3265 (2003).

[23] Y. T. Li et al., Phys. Rev. Lett. 96, 165003 (2006).

[24] S. Bastiani, A. Rousse, J. P. Geindre, P. Audebart, C. Quoix, G. Hamoniaux, A. Antonetti, and J.-C. Gauthier, Phys. Rev. E 56, 7179 (1997).

[25] Y. T. Li et al., Phys. Rev. E 69, 036405 (2004).

[26] H. Ruhl, Y. Sentoku, K. Mima, K. A. Tanaka, and R. Kodama, Phys. Rev. Lett. 82, 743 (1999).

[27] Y. Sentoku et al., Phys. Plasmas 6, 2855 (1999).

[28] D. F. Cai, Y. Q. Gu, Z. J. Zheng, W. M. Zhou, X. D. Yang, C. Y. Jiao, H. Chen, T. S. Wen, and S. T. Chunyu, Phys. Rev. E 70, 066410 (2004).

[29] D. Duston, J. E. Rogerson, J. Davis, and H. Blaha, Phys. Rev. A 28, 2968 (1983).

[30] J. Scofield, Phys. Rev. A 9, 1041 (1974).

[31] P. Mora, Phys. Rev. Lett. 90, 185002 (2003).

[32] NRL Plasma Formulary, Washington, DC, 1990, NRL/PU/ 6790-00-426.

[33] H.-K. Chung et al., High Energy Density Physics 1, 3 (2005). 